\title{
Los traslados de ciudades en América: autorretrato de una sociedad en crisis
}

\author{
Alain Musset \\ Ecole des Hautes Études en Sciences Sociales-Paris
}

\begin{abstract}
Entre el principio del siglo XVI y el final de la época colonial, varias decenas de ciudades fundadas en América por los conquistadores españoles se cambiaron de lugar. Las causas de estos traslados variaban y podían combinarse: clima malsano, desastres naturales, ataques de indios o piratas, cambios de eje económico... El análisis de este momento de crisis aguda valoriza las contradicciones internas de la sociedad colonial ya que la decisión de la mudanza no era siempre fácil de tomar, sobre todo cuando la ciudad había tenido tiempo de desarrollarse y de arraigarse en su territorio. Mientras que los distintos grupos de presión se enfrentaban para obtener su desplazamiento o para mantenerse en el sitio antiguo, las solidaridades de fachada se borraban ante las profundas grietas que cruzaban a una sociedad urbana desestabilizada y en búsqueda de una nueva legitimidad política. Incluso se iba a poner en tela de juicio el papel jugado por el Rey, y el concepto polisémico de Patria comenzaba a tomar un nuevo sentido para los criollos que se consideraban perjudicados por los representantes de la Corona.
\end{abstract}

Palabras claves: América hispanica, traslados de ciudades, sociedad urbana, crisis politica, Patria.

Between the beginning of the XVIth century and the end of the colonial time, tens of cities founded in America by the Spanish conquerors were changed of place. The causes of these transfers were varied and could be combined: unhealthy climate, natural disasters, attacks of Indians or pirates, changes of economic axis... The analisis of this moment of acute crisis emphasizes internal contradictions of the colonial society because the decision of the transfer was not always easy to take, especially when the city had time to grow and root in its territory. Whereas the various lobbies clash to obtain their displacement or their maintenance in the place, apparent solidarity is disappearing in front of deep cleavages which cross a destabilized urban society in search of a new political legitimacy. Even the role of the king is then criticized and the polysemous concept of Fatherland starts to take a new direction for Creoles who estimate themselves injured by the representatives of the Crown.

KEYWORDS: Spanish America, city transfers, urban society, political crisis, fatherland.

La ville, avec ses constructions de pierre, ses fondations, ses lieux de pouvoir et de culte, semble être bâtie pour durer, pour assurer l'enracinement des sociétés dans l'espace et dans le temps. Pourtant, entre le début 
de la conquête du continent américain et la fin de l'époque coloniale, les villes fondées puis déplacées par les Espagnols se comptent par dizaines. $\mathrm{Si}$ les attaques d'Indiens hostiles ont provoqué la majorité des transferts réalisés au cours du XVIe siècle, au fil du temps ce sont les conditions naturelles qui ont poussé les conquérants à chercher un meilleur site. Éruptions volcaniques, coulées de boue mais surtout tremblements de terre ont joué un rôle central dans cette histoire marquée par les désastres: si le cas de Guatemala est le plus connu, le même sort a touché Santiago de los Caballeros et Concepción de la Vega (République dominicaine, 1562), Léon (Nicaragua, 1610), La Concepción de Chile (1751), Chillan (Chili, 1751-1835), Riobamba (Équateur, 1797) - parmi tant d'autres. À l'issu de sept années de recherche sur le terrain et dans les archives, j'ai ainsi compté plus de 160 centres urbains qui ont changé au moins une fois d'emplacement au cours de cette période, sans compter ceux qui ont subi le même sort au XIX ${ }^{e}$ et au XX ${ }^{e}$ siècle, comme San Juan Parangaricutiro au Mexique ou Baeza et Pelileo en Équateur. ${ }^{1}$ Or, même si le nombre des cas relevés est impressionnant, l'ensemble ne prétend pas être exhaustif. Aucun catalogue ne pourra jamais recenser toutes les villes qui, au cours des cinq derniers siècles, ont été déplacées sur un territoire aussi vaste que celui de l'ancien empire espagnol d'Amérique.

Le déplacement des villes occupe donc une place fondamentale, mais encore peu et mal étudiée, dans la construction de l'espace hispano-américain. Travailler sur ce thème permet non seulement de s'interroger sur les conceptions urbaines des fondateurs, mais aussi de réfléchir sur les formes et les fonctions de la cité et sur les relations souvent conflictuelles qu'elle entretient avec son environnement. En outre, l'analyse d'un tel moment de crise met en valeur les contradictions internes de la société coloniale, car la décision du transfert n'est pas toujours facile à prendre, surtout quand la ville a eu le temps de se développer et de s'enraciner dans son territoire. Un tel dilemme séparait généralement la communauté en deux camps (partisans et adversaires du transfert), comme le montrent les grands débats organisés en 1717 à Guatemala, lors des réunions du Conseil municipal élargi à l'ensemble de la population, ou ceux de la controverse de 1773, quand la Couronne imposa finalement l'évacuation de la Ciudad de Santiago. On retrouve les mêmes schémas, compliqués par les rivalités personnelles et les querelles politiques ou religieuses, dans presque tous les projets de

1 Musset, Alain: Villes nomades du Nouveau Monde. Éditions de l'EHESS, Paris, 2002. 
déplacement. Il s'agit donc d'un moment clé de l'histoire de la ville hispano-américaine, puisque les solidarités de façade qui structuraient la communauté s'effacent devant les profonds clivages de la vie coloniale. Or, quand la crise urbaine déclenche une crise politique, ce sont les fondements de la civilisation espagnole qui sont menacés car, selon le modèle hérité de l'époque romaine, l' "urbs", la ville matérielle, risque de ne plus correspondre à la "civitas", l'assemblée des citoyens qui constitue l'état.

\section{Les divisions internes: partisans et adversaires du transfert}

Dans les premières années de la conquête, quand les Espagnols n'avaient pas encore enraciné leurs établissements, la décision pouvait être prise à l'unanimité des habitants. En revanche, dès que la cité prenait de l'importance et que les groupes sociaux se distinguaient les uns des autres, il devenait de plus en plus difficile de faire coïncider toutes les opinions et de prendre, sans attiser de graves conflits internes, les mesures qui s'imposaient aux yeux de certains, mais pas à l'ensemble de la communauté. Les adversaires du projet de transfert faisaient alors de la résistance et tentaient par tous les moyens de se maintenir dans les lieux, en dépit des recommandations, puis des ordres venus d'Espagne.

Dans ce contexte, toutes les ruses étaient bonnes pour éviter le déplacement, même quand la décision avait été prise par le roi lui-même. C'est ainsi que les habitants de Veracruz hostiles au transfert vers la plage de Buytron (site actuel de la ville) envoyèrent à la Couronne une lettre pour démontrer tous les inconvénients du nouveau site: un lieu désert et désolé, envahi par les sables, sans herbe, sans bois, sans eau, mis à part un marécage malsain tout juste bon à abreuver le bétail. Les auteurs de la lettre se doutaient cependant que leurs arguments médico-géographiques avaient peu de chance d'attendrir les membres du Conseil des Indes. C'est pourquoi ils sortirent de leur manche un atout imparable: à une lieue de la plage de Buytron, s'élevait l'île des Sacrifices qui pouvait offrir un refuge sûr à toute une flotte de pirates. De nuit, ceux-ci risquaient d'attaquer le village, de brûler toutes les maisons et de s'emparer du trésor royal avant même que les troupes installées dans la forteresse de San Juan de Ulua puissent réagir. Les opposants au transfert avaient su toucher la corde sensible de la Couronne. Ils marquèrent donc des points et obtinrent de retarder l'échéance fatale puisque, dans une cédule royale datée du 13 juin 1599, Philippe 
III écrivit aux autorités locales de conserver le trésor à Veracruz jusqu'à ce que la nouvelle ville soit bâtie, fortifiée, et qu'une population nombreuse puisse en garantir la sécurité.

Dans le cas du transfert de Santa María de la Victoria (Tabasco, Mexique), approuvé en 1619 par le vice-roi marquis de Guadalcazar, les habitants de la ville détruite par les pirates anglais et hollandais furent l'enjeu d'une lutte acharnée entre les autorités civiles, favorables au déplacement, et la hiérarchie catholique, hostile à l'abandon de la cité. C'est sans doute à cause de ces résistances internes que l'Alcalde mayor de la province, don Fernando Martínez de Leyva, n'accorda qu'un mois de délai aux habitants de Santa María de la Victoria, qu'ils soient espagnols, indiens, noirs ou mulâtres, pour abandonner le site ancien de la ville et rejoindre le site de Villahermosa. ${ }^{3}$ Le délai fut jugé beaucoup trop court, notamment par ceux qui s'opposaient de manière plus ou moins ouverte au transfert et qui invoquaient des raisons personnelles pour retarder l'échéance. C'est le cas de Martín Nieto qui, tout en se déclarant prêt à abandonner sa maison, réclama plus de temps pour réaliser le déménagement qu'on lui demandait, car il devait non seulement faire construire une nouvelle demeure pour sa femme et ses enfants, mais aussi dépiquer les quatre-vingt fanègues de maïs de sa dernière récolte. Sa demande fut sèchement refusée par l'alcalde mayor, convaincu du fait que le dépiquage des épis de maïs n'était qu'un fallacieux prétexte utilisé par Nieto pour désobéir aux ordres du vice-roi.

\section{Clivages sociaux et fractures politiques}

Quand la période de crise atteignait son point culminant, l'unité de façade qui permettait de masquer les dissensions internes de la société coloniale volait en éclats. Religieux réguliers et séculiers se déchiraient à belles dents. Créoles et Espagnols de la péninsule ressortaient leurs vieilles rancœurs et exprimaient au grand jour d'inextinguibles jalousies. Riches et pauvres se dressaient les uns contre les autres. Chacun invoquait la légitimité de sa naissance, de son rang, de ses racines, pour justifier son choix en faveur du transfert ou sa volonté de rester dans la ville en ruine.

2 Archivo General de la Nación, México, Reales cédulas duplicados, vol. 1 bis, 29, 27.

3 Izquierdo, Ana Luisa: El abandono de santa María de la Victoria y la fundación de San Juan Bautista de Villahermosa, UNAM, México, 1995, pág. 97. 
L'administration locale était critiquée. Le roi lui-même était remis en cause. On lui reprochait de ne pas être là, de ne pas partager la souffrance de ses malheureux sujets, de ne pas comprendre leur douleur. Ses représentants, quel que fût leur statut (vice-roi, capitaine général, gouverneur...), devaient gérer une situation explosive, qui risquait de dégénérer en conflit ouvert, parfois même en guerre civile. Il fallait donc pouvoir trancher sans provoquer de blessures irrémédiables dans le corps social, alors que chaque parti semblait prêt à en découdre, que les divisions l'emportaient sur l'esprit de groupe et que l'on soupçonnait toujours les Indiens de vouloir profiter d'un moment d'égarement pour prendre leur revanche sur des conquérants affaiblis.

Le cas de San Bartolomé de Chillán (Chili) est à cet égard particulièrement révélateur. Fondée en 1580 sur les bords de la rivière du même nom, cette petite ville devait permettre aux Espagnols de coloniser des terres quotidiennement menacées par les guerriers mapuches, longtemps considérés comme indomptables, et de garantir la sécurité des routes commerciales entre Santiago et les villes du sud - principalement la Concepción. À peine sortie de terre, en 1588, la localité était attaquée par des Indiens insoumis. Dix ans plus tard, au cours de la grande insurrection qui faillit mettre un terme définitif à la présence espagnole dans tout le sud du Chili, San Bartolomé était réduite en cendres. En 1628 et en 1629, puis en 1655, de nouveaux raids mapuches faillirent provoquer la disparition de la ville martyre. Entre temps, ses habitants avaient subi deux séismes destructeurs (1647 et 1655), ainsi que des inondations répétées. La plus grande partie de la population avait alors abandonné les lieux, en quête d'une terre moins hostile.

En 1664, à l'initiative d'Angel de Peredo, gouverneur du Chili, la ville fut déplacée sur la rive droite du Rio Chillán (aujourd'hui Rio Viejo), dans la partie basse de l'actuelle municipalité de Chillán Viejo (carte $\mathrm{n}^{\circ} 1$ ). On espérait ainsi redonner vie à un élément important du système de défense mis en place par les conquérants afin de faire barrage aux incursions des Mapuches. En 1751, un tremblement de terre dévastateur fut à l'origine du deuxième déplacement de San Bartolomé. Cependant, alors que le premier transfert ne semble pas avoir suscité de graves dissensions internes, le second révéla de réelles divergences entre les principaux acteurs de la vie urbaine. Dans un premier temps, tout le monde s'accorda pour reconnaître l'ampleur des dégâts causés par les violentes secousses du 25 mai 1751: des maisons entières s'étaient effondrées; de larges et profondes failles 


\section{ALAIN MUSSET}

entaillaient les murs les plus épais des églises et des couvents; le sol était littéralement déchiré par des crevasses qui s'ouvraient comme des pièges sous le pied des passants. ${ }^{4}$ Très vite, des voix s'élevèrent pour réclamer le déplacement de la communauté vers le site de la Horca, qui se trouvait sur une hauteur, à moins de cinq cents mètres au nord de la ville en ruine. Pour conserver dans la région de Nuble un point d'ancrage fort, il s'agissait de maintenir la présence d'une puissante garnison espagnole, mais aussi d'éloigner les habitants d'un cours d'eau aux crues brutales et dangereuses.

\section{Carte n. ${ }^{\circ}$ 1: les déplacements de la ville de Chillán}

Le 7 août 1751, un conseil municipal ouvert à l'ensemble de la population fut organisé par les autorités locales, avec l'accord du gouverneur et capitaine général du Chili, Domingo Ortiz de Rosas, afin d'avaliser le choix du site de la Horca, sur lequel on avait déjà commencé à édifier les premiers bâtiments de la nouvelle Chillán. En effet, dès le début du mois de juillet, une quarantaine de cabanes et une chapelle, bâties à la hâte en prévision de l'hiver, abritaient une partie de la population, chassée de ses pénates par le séisme. Pour les autorités locales, il s'agissait d'assurer la cohésion de la communauté et d'apaiser les rancœurs qui pourraient naître des dissensions entre les habitants. ${ }^{5}$ Cependant, ce cabildo abierto fut surtout l'occasion pour les opposants au transfert de se manifester, par écrit tout d'abord, puis en prenant publiquement la parole.

Les fractures internes de la société locale apparaissent alors au grand jour: tous unis pour lutter contre les Indiens, les quelque mille cinq cents habitants de Chillán appartiennent de fait à des groupes sociaux, politiques et économiques aux intérêts divergents. L'un des principaux opposants au transfert est le propre procurador de la ville, Carlos de Acuña y Salinas, qui se présente comme le porte-parole des ordres religieux et de la plus grande partie des habitants, mais surtout des pauvres. ${ }^{6}$ D'après lui, le site choisi manquait d'eau et les terrains agricoles n'étaient pas aussi fertiles qu'au bord de la rivière. En outre le sol de la Horca était infesté de sauterelles et d'insectes nuisibles qui risquaient d'envahir tous les jardins. Enfin, rares

4 Archivo Histórico de Chile (en adelante AHC), Santiago de Chile, Capitanía general, 983, 17 514, 224-226.

5 Ibídem, 244

6 Ibídem, 236 
étaient les habitants qui pouvaient faire face au coût financier d'un transfert impliquant la reconstruction d'une maison, la mise en valeur des terres cultivables et la plantation des vignes et des arbres fruitiers qui avaient fait la richesse de la ville. Dans une deuxième lettre, le même Carlos de Acuña, se présente surtout comme l'avocat des franciscains et des dominicains, dont les intérêts étaient directement menacés par le nouveau projet urbain. En effet, pour alimenter en eau potable la population, il fallait utiliser celle qui faisait tourner deux moulins appartenant aux dignes religieux et dont la valeur était estimée respectivement à six cents et huit cents pesos.

Le cabildo abierto du 7 août fait apparaître clairement les lignes de force qui traversent le petit monde de Chillán. Deux camps se font face: les adversaires et les partisans du déplacement vers le site de la Horca. Dans le premier, on trouve, bien entendu, les franciscains et les dominicains, qui minimisent les problèmes posés par l'ancien site (humidité, risque d'inondation) et soulignent qu'ils risquent de perdre les revenus tirés de l'exploitation de leurs moulins (trente pesos par an pour le couvent de Saint Dominique). Par solidarité, les frères de la Merced se rangent de leur côté, même s'ils reconnaissent avoir souffert à plusieurs reprises des inondations provoquées par la rivière proche. En revanche, comme souvent, les jésuites font bande à part parmi les membres du clergé régulier. Fidèles à leur réputation, c'est avec méthode qu'ils rejettent le site ancien de la ville. Leurs arguments se fondent sur des traditions scientifiques depuis longtemps éprouvées: "Dicho paraje tiene contra el los tres elementos del agua, la tierra, y el ayre; el del agua por lo amenasada que se halla del rio; la tierra por que en ella hazen mas operazion los temblores, como se ha visto; y el ayre por lo inficionado que corre en dicho paraje por cuyo motivo es poco sanoy se experimentan muchas enfermedades" (ibidem, f. 247 v.). ${ }^{7}$ Les membres de la compagnie de Jésus insistent par ailleurs lourdement sur le fait qu'il serait facile d'alimenter en eau la nouvelle localité. Cette remarque n'est pas le fruit du hasard car, sous prétexte de s'incliner devant le bien public, ils soutiennent un projet susceptible d'affaiblir la position de leurs concurrents franciscains et dominicains, menacés de perdre leurs moulins et donc une partie de leurs ressources financières. Une alliance stratégique est donc conclue avec le curé de la paroisse, Simón de Mandiola, pour qui le déplacement de Chillán est aussi l'occasion de régler ses comptes avec le clergé régulier.

7 Ibídem, 247 v. 
Outre les représentants de l'Église et du conseil municipal, cinquante vecinos s'expriment librement sur cette question. Le oui l'emporte sans contestation possible, puisque seules trois voix soutiennent la position des franciscains et des dominicains. Les autres suivent l'avis des membres du conseil municipal et surtout des jésuites, dont on admire de manière presque unanime la rigueur de la démonstration et la justesse des propos. Même les plus anciens, ceux qui se targuent d'avoir toujours vécu dans la ville aujourd'hui en ruine et d'avoir assumé de nombreuses charges municipales se déclarent convaincus par les "puissants raisonnements" des disciples de Saint Ignace. Mieux encore, les propriétaires fonciers se disent prêts à abandonner leurs terrains pour s'installer sur le site de la Horca. La cause est donc entendue: l'union des membres du conseil municipal, des jésuites, du clergé séculier et des propriétaires terriens a fait pencher la balance en faveur du transfert, au détriment du clergé régulier et des "pauvres" — cette catégorie sociale mal définie- dont le procurador Carlos de Acuña prétendait défendre les intérêts.

Mais l'histoire de Chillán ne s'arrête pas avec le déplacement de la vieille ville vers le site de la Horca. En février 1835, un nouveau tremblement de terre détruisit la malheureuse cité et le projet d'un troisième déplacement (décrété le 5 novembre de la même année), suscita de vifs débats dans la population (14 576 personnes recensées en 1812): les blessures ouvertes par le précédent transfert n'étaient pas encore refermées qu'il fallait à nouveau organiser le départ et le relogement des habitants sinistrés. Surprises par l'ampleur des mouvements de résistance, les autorités chiliennes décidèrent de ne brusquer personne: dans un pays qui venait à peine d'obtenir son indépendance, on ne pouvait plus agir comme au temps de la Couronne espagnole. Ceux qui voulaient rester dans la ville en ruine pouvaient le faire. Les autres étaient cordialement invités à se rendre sur le nouveau site choisi par les experts.

Après la proclamation officielle du transfert, la municipalité de Chillán fut partagée en deux entités distinctes: Chillán Viejo (site de la Horca) et Chillán Nuevo (nouveau site). Très tôt, les habitants du Viejo Chillán se plaignirent du régime de faveur accordé par les autorités de la province à ceux qui s'étaient installés dans la nouvelle ville, et des mauvais traitements qui leur étaient infligés pour les décourager de vivre dans leurs anciennes demeures. C'est ainsi que des mesures furent prises par le conseil municipal, installé sur le nouveau site, pour limiter les possibilités de vendre et d'acheter des marchandises dans les boutiques et sur le marché 
de la vieille ville. De la même manière, les échevins refusèrent de bâtir une école primaire pour les enfants des personnes restées sur place. En revanche, ils autorisèrent l'abattage du bétail dans les rues et sur la place principale de Chillán Viejo, afin d'augmenter les désagréments de la population.

Dans une lettre datée (semble-t-il), du 21 novembre 1840, les représentants de la communauté protestèrent parce que l'intendant avait ordonné la destruction des églises de Santo Domingo et de la Merced, mais aussi du marché, sous prétexte que tous ces édifices, durement touchés par le séisme, menaçaient de s'effondrer sur la tête des fidèles, des commerçants et de leur clientèle: "Nosotros, y todos los demás habitantes de la antigua población de Chillán, nos hallamos sufriendo las persecuciones qe hasta los tiempos modernos havían sido exclusivas a la desgraciada tribu de Jacob. No exageramos por cosa alguna al haser esta aplicación, y aún tal ves no es exacta por diminuta, porque al fin, en la suabidad de costumbres e ilustración del siglo actual, han encontrado los israelitas bastante hospitalidad en Europa para fundar sus sinagogas y celevrar sus juntas religiosas; pero aquí se nos destruyen nuestras iglesias por orden de la intendencia". ${ }^{8}$

Qualifiés de "rebeldes" et d' "obstinados" par les services de l'Intendance, les habitants du Viejo Chillán se considéraient quant à eux comme "perseguidos", et accusaient les artisans de la nouvelle ville de n'être qu'une bande d'infâmes spéculateurs. L'un des plus ardents défenseurs de l'ancien site, Juan Antonio Zuñiga, inonda de lettres les services municipaux et ceux de l'Intendance, pour réclamer un traitement équitable entre les deux parties de la ville. Cet acharnement lui valut d'ailleurs des poursuites judiciaires et une peine de prison. Quelques mois à peine après la prise de décision du transfert, il écrivit une requête à l'intendant de la province pour protester contre le choix du nouveau site, qu'il estimait humide et malsain. ${ }^{9}$ D'après lui, la majorité des habitants s'opposait à cette décision et réclamait leur maintien dans les lieux. En 1848, les représentants du Viejo Chillán manifestaient toujours les mêmes rancœurs vis à vis de ceux qui avaient abandonné leurs antiques demeures, trahissant ainsi la mémoire des fondateurs de la cité: "Y todo ello debido al odio, y encarnisamiento de las pasiones de los pobladores de la nueva ciudad, y sus autoridades" ${ }^{10}$ Ils reprochent aux membres du conseil munici-

8 AHC, Santiago de Chile, , 257, 180, 370.

9 Ibídem, 378.

10 Ibídem, 354. 
pal de tous loger dans la partie neuve de la ville et de ne défendre les intérêts que de leurs proches voisins. Or, c'est dans la partie ancienne que la croissance démographique se révélait la plus forte, car les terrains étaient moins chers et attiraient de nombreux migrants venus de la campagne. D'après leurs comptes, il restait 106 maisons habitables après le tremblement de terre de 1835. Treize ans plus tard, on en dénombrait 903, dont 481 étaient bâties en matériaux durables. Elles abritaient une population estimée à quelque huit mille âmes.

Pour résoudre le problème et apaiser les tensions, les délégués de la vieille ville préconisèrent tout un train de mesures destinées à réduire les inégalités de traitement entre les deux parties de l'agglomération. Il s'agissait tout d'abord d'assurer le nettoyage des rues et d'accroître la surveillance policière du Viejo Chillán, car l'arrivée de nouveaux migrants avait provoqué une forte augmentation de l'insécurité. Pour cela, on réclamait la présence d'un caporal et de quatre soldats. Ensuite, il apparaissait nécessaire de fonder une paroisse séparée de la ville nouvelle, pour permettre aux habitants de suivre la messe et d'assister aux cérémonies religieuses sans avoir à accomplir un long et pénible trajet. Mais cette suggestion ne servait qu'à enrober de manière élégante la question centrale: on demandait au pouvoir politique de créer une commune indépendante, en confirmant sur le plan juridique l'existence de deux villes distinctes, Chillán et Chillán Viejo. Comme le disaient les auteurs de la pétition: "Pues ocho mil y mas almas, que viven en novecientas y tres casas no puedan estar privada de este bien. Hay una infinidad de pueblos en la republica, que con una tercera parte menos de poblacion que la que tiene hoy la ciudad antigua de Chillan son representatas por municipalidad"."

De guerre lasse, la République finit par céder aux instances des habitants de Chillán Viejo et, en 1925, leur accorda une entière autonomie. Pourtant, deux ans plus tard, les deux villes furent à nouveau réunies pour ne former qu'une seule municipalité, dont le siège se trouvait dans la ville neuve. La tension entre les deux communautés fut alors ravivée et une longue période de troubles et de rivalités internes s'ensuivit. L'épilogue de cette longue histoire se situe presque soixante-dix ans plus tard, quand la loi n 19434 du 22 décembre 1995 consacra le rétablissement de la municipalité de Chillán Viejo, au détriment de la municipalité de Chillán, amputée de toute la partie sud de son territoire.

11 Ibídem, $355 \mathrm{v}$. 


\section{Débats passionnés et comptes d'apothicaires}

Comme on l'a vu, le choix du transfert engageait donc tout l'avenir de la communauté et risquait de remettre en cause les fondements de la société urbaine. À elle seule, la perspective d'une redistribution de la carte foncière pouvait mettre en péril l'ordre établi. Elle menaçait la hiérarchie économique et sociale matérialisée (entre autres) par l'emplacement de tous les édifices, publics ou privés, qui marquaient l'espace de la ville ancienne. Surtout, elle remettait en cause l'unité de façade d'une communauté qui, pour faire front commun contre les Indiens, avait besoin d'affirmer son identité collective et de multiplier les signes de cohésion.

Conservée à la Bibliothèque nationale de Madrid une Breve y verdadera noticia de los trabajos que con el incendio del volcán, terremotos que se le siguieron y otras consecuencias, ha padecido esta ciudad de Santiago de los Caballeros de Guatemala, desde el día 27 de agosto de este año de 1717 hasta el día de agosto de 1718, fait le point sur les dégâts provoqués par le séisme et évoque de manière détaillée les débats qui ont opposé entre eux les habitants de la ville sur le projet de transfert. ${ }^{12}$ On y voit que, derrière les discours convenus et les traditionnelles formules de rhétorique, chaque groupe défend non seulement ses intérêts, mais aussi sa vision du monde et sa conception de la vie urbaine. Ce document, rédigé par un partisan du maintien dans les lieux, laisse néanmoins la parole à ses adversaires, pour mieux les confondre. Il démonte leur stratégie, qui consiste à exagérer les dégâts et à prédire la destruction complète de la cité dans des discours dignes de l'apocalypse: la ville va être engloutie par les volcans qui l'entourent et il ne restera de la vallée qu'une vaste étendue d'eau et de boue là où se dressaient les clochers des églises. Les partisans du traslado tentent d'imposer la politique du fait accompli, en incitant les habitants à quitter la ville pour que les autorités enregistrent officiellement l'abandon du site. C'est le début d'une bataille de procédure qui met en jeu la notion même de cité, de communauté, de "république", pour reprendre l'expression utilisée par l'auteur du rapport:

"Muy grandes estragos hicieron los terremotos en los edificios, mas solo tocaron a la ropa de la ciudad, le despedazaron el vestido y le deslizaron su adorno. Mucho mayor daño causó la dispersión de sus vecinos, pues esto fue despedazarle sus mismo cuerpo y arrojar sus miembros por varias y distantes partes [...]. La deserción que hicieron desta ciudad sus vecinos el día 30 de septiembre fue muy grande, pues salió la maior

12 Biblioteca Nacional, Madrid, Ms 3534. 
parte de la plebe, y otros muchos caballeros y personas de categoría, pero todavía quedaron en la ciudad algunos caballeros republicanos, personas honradas, eclesiásticos seculares y lo mas de los regulares, y gran parte de la plebe, que se restituyó luego incontinente que paso el día que hicieron tan horroso los falsos agüeros, que fue el día 4 de octubre, todo bastante y sobrado para constituir ciudad y así nunca ya pudo dar por totalmente desierta; ademas que la ciudad se entiende desierta por la total deserción que hacen los vecinos, libre y voluntariamente, con ánimo de no volver mas a ella, no por la deserción que aquí hicieron, violentados y engañados con la fiera e abusiva sentencia de que dentro de dos horas se había de hundir y anegar la ciudad, por las cuales voces salieron huyendo, dejando sus bienes, con ánimo de volverse a sus casas luego que pasase el termino peremptorio señalado a la fingida submersion". ${ }^{13}$

Le 6 octobre 1717, une assemblée générale de tous les citoyens de Santiago de Guatemala permit à chaque groupe d'exposer ses arguments, pour ou contre le transfert. À cette occasion, tous les clivages de la société coloniale, masqués par l'unité affichée d'un monde à la fois urbain et espagnol, qui s'opposait aux campagnes indigènes, apparurent au grand jour. Les conventions politiques s'effacèrent, les liens traditionnels de solidarité, de clientélisme ou de soumission furent remis en cause. Les membres du Conseil municipal, partisans du transfert, n'hésitèrent pas à s'en prendre directement aux hommes d'Église qui, pour préserver leurs seuls intérêts, refusaient de quitter la ville détruite. Dans leur lettre du 8 octobre 1717 , ils exprimaient clairement leur opinion sur ce thème, le ramenant à son point de départ - l'argent - occulté par de grands discours sur le devoir qui s'imposait aux prêtres de préserver l'union d'une communauté frappée par le malheur: "pues los religiosos han de perder sus rentas, los clérigos sus capellanías, los conventos sus posseciones". ${ }^{14}$

Mais les divisions internes suivaient plusieurs lignes de fracture qui ne recoupaient pas toujours la seule opposition entre civils et religieux. Les opposants au transfert de Guatemala rappelèrent qu'une partie des échevins et des habitants avaient essayé d'imposer par la force, l'arme au poing, le départ des autres citadins, en profitant du trouble provoqué par la destruction des principaux édifices de la ville. Ils leur opposèrent alors le cas d'autres localités touchées par de violents séismes et dont le déplacement n'avait jamais été décidé car les tremblements de terre font partie de la nature même du continent américain: Lima, Quito, Truxillo, Oaxaca, Puebla, Panamá, et d'autres encore. ${ }^{15} \mathrm{Il}$ ne servirait donc à rien de fonder quelques

13 Biblioteca nacional, Madrid, Ms 3 534, 55. y 59.

14 Archivo General de Centro-América, Guatemala, A1. 10, 2 273, 16 495, $40 \mathrm{v}$.

15 Biblioteca Nacional, Madrid, Ms 3 534f. 56-56 v. 
kilomètres plus loin une nouvelle ville qui serait tout autant que l'ancienne exposée aux convulsions de la croûte terrestre.

Le débat organisé entre les deux groupes ne fit que révéler au grand jour les discordes et les clivages d'une société profondément divisée sur un thème aussi important. Le neuf octobre, jour dédié à Saint Denis "avocat des tremblements de terre et saint patron de la ville", un nouveau séisme secoua la cité, provoquant de nouveaux mouvements de panique. Le quinze octobre, les résultats de la consultation furent proclamés. Dans le clan des opposants, on trouvait les dominicains, les jésuites, les missionnaires du collège apostolique et les religieuses du couvent de Sainte Thérèse. Parmi les partisans du transfert, outre les voix de la majorité des vecinos, on comptait celles de l'évêque, du conseil municipal et des alcades ordinaires, des députés du commun et des autres ordres religieux (Saint François, la Merced, la Concepción et Sainte Catherine - malgré l'opposition de la mère supérieure). La situation était d'autant plus complexe que l'Église ne parvenait pas à offrir un front uni et que ses membres, pour attirer la conviction des indécis, essayaient tour à tour de se présenter comme les meilleurs interprètes de l'orthodoxie catholique.

Il fallait donc éviter d'être pris en défaut par des arguments imparables, qui pouvaient remettre en cause la légitimité théologique des participants au débat. Au Guatemala, pour la première fois, science et religion se trouvaient face à face. De manière assez perfide, les opposants au projet de transfert s'interrogèrent sur la qualité des sentiments religieux de leurs adversaires, qui pensaient échapper à la colère divine en fuyant leurs responsabilités d'hommes et de pécheurs. Pour eux, la meilleure façon de lutter contre les séismes était de demander grâce au Seigneur. Les partisans du transfert furent bien obligés de concéder qu'on ne pouvait pas esquiver un juste châtiment en changeant simplement de place. Ils soulignèrent cependant qu'il fallait distinguer deux types de mécanisme dans les actions du Créateur: s'il était impossible d'aller à l'encontre de sa volonté directe, on pouvait néanmoins tenter d'infléchir certains effets secondaires de son courroux, comme l'étaient les tremblements de terre. Par ailleurs, comme seul un miracle pouvait sauver les hommes en cas de cataclysme, il était souvent préférable d'utiliser un "moyen naturel", la fuite, plutôt que d'attendre un pardon qu'il n'était pas toujours facile de mériter. ${ }^{16}$

16 Archivo Histórico Nacional, (en adelante AHN) Madrid, Diversos, Documentos de Indias, $419(5), .15 \mathrm{v}$. 
Pour corser les débats, partisans et adversaires du transfert mettaient en avant des arguments d'ordre économique tout aussi discutables: le coût comparé du transfert ou de la reconstruction. En effet, bâtir une nouvelle ville impliquait des dépenses que de nombreux habitants n'étaient pas prêts à assumer, préférant réparer les dégâts provoqués par les pirates ou les secousses sismiques. Ainsi, en 1562, l'auteur d'un premier projet de transfert de Veracruz évoquait l'opposition probable des habitants et cherchait à limiter par avance les frais susceptibles d'être engagés par la Couronne: "que V.M. mande que los vecinos de la ciudad de la Veracruz se pasen al sitio nuevo que trazó el bachiller Martínez, en el hato que llaman de doña María, una legua de donde están ahora. El impedimiento que hay, es que dicen que tienen sus casas hechas, y que les dan de comer, que les ayuden para hacerlas acullá [sic]; con pagarles la mitad de lo que valen sus casas, luego se pasará. Y esto justo es que V.M. los ayude y favorezca, pues que todo se cae en casa, y con menos de cincuenta mil pesos se pagaran las casas, segun dicen". ${ }^{17}$

Ce sont les mêmes raisons de trésorerie qui ont conduit les autorités de Guatemala à réparer les dégâts du tremblement de terre de 1717 plutôt que de rebâtir la ville sur des bases plus saines et dans un site jugé moins exposé. Pourtant, ces travaux ont coûté à eux seuls des sommes colossales: une évaluation réalisée à l'époque fixait à 355300 pesos les frais occasionnés pour la réhabilitation des églises et des couvents de la ville, dont quatre-vingt mille pesos pour celui de la Merced, quarante-six mille pour celui de San Francisco et trente-huit mille pour celui des Augustins (AGCA, A1. 10, leg. 2 273, exp. 16 495, f. 56 r.-60 v). À ces dépenses rondelettes, il fallait ajouter 10750 pesos pour réparer la mairie, la prison, les quatre boucheries et les aqueducs qui alimentaient la ville en eau potable. L'ampleur et la fréquence des tremblements de terre conduisirent les échevins à protester contre le maintien de la ville dans une région trop dangereuse. Une lettre du Conseil municipal, adressée au Conseil des Indes et datée du 8 octobre 1717, manifesta l'impatience des édiles, même s'ils finirent (au moins provisoirement) par renoncer à leur projet de déplacement : "No ai caudales que basten a que cada veinte o veinte y cinco años sea preciso como lo ha mostrado la experiencia reedificar el medio o el tercio del lugar sino todo como lo presente de que resulta que manteniendonos en este parage, cada cinquenta o sesenta años ha menester la ciudad de nuevo

17 AHN, Madrid, Diversos, Documentos de Indias, 184. 
levantarla, por que ahora que esta arruinado mas de medio lugar sera bienque lo que se ha de gastar aqui, quien lo pudiere hazer, lo vaya a gastar a otra parte con la esperanza de que les dure maiormente". ${ }^{18}$

\section{Ville créole et couronne espagnole}

Si le moment de crise que représente la décision du transfert permet de mettre en valeur les dissensions internes de la société coloniale, il sert aussi de révélateur aux fractures qui s'agrandissent au fil du temps entre l'administration espagnole et la société créole. Les représentants du roi, ballottés d'un poste à l'autre et dont la seule ambition était de s'élever dans la hiérarchie des honneurs administratifs, ne pouvaient pas raisonner comme leurs administrés, chez qui l'attachement au sol natal prenait une place de plus en plus importante. En résistant aux pressions de la Couronne, et surtout aux ordres de ses délégués (quel que soit leur niveau d'intervention), les créoles exprimaient de manière souvent confuse une revendication identitaire qui, à l'aube du XIX ${ }^{\mathrm{e}}$ siècle, finirait par éclater au grand jour.

\section{Une ville contre son roi: l'échec du déplacement de Mexico}

Dans ce contexte, le cas de México prend valeur d'exemple et de symbole. En effet, dans la capitale de la Nouvelle-Espagne, les tensions se sont lentement accumulées entre les Espagnols fraîchement débarqués et les créoles qui se sentaient exclus des cercles du pouvoir. Le désastre provoqué par la grande inondation de 1629 fut l'occasion pour les échevins de Mexico de relever la tête et de se poser en victimes de l'administration espagnole: alors que la Couronne voulait imposer le déplacement de la ville, ses habitants refusèrent d'abandonner le site choisi par Hernán Cortés. Une telle résistance s'explique autant par leur crainte de perdre de grandes sommes d'argent que par leur désir de ne pas abandonner à la nature et aux Indiens la capitale d'un royaume dont ils occupaient le sommet.

Le 29 avril 1631, le conseil d'État se réunit à la demande du roi pour statuer sur le sort de la ville qui, depuis presque deux ans, vivait sous les eaux Autour du cardinal Zapata, on retrouvait quelques-uns des plus

18 AGCA, Guatemala, A1. 10, 2 273, 16 495, 41. 


\section{ALAIN MUSSET}

importants personnages du royaume: le comte d'Oñate, le marquis de Gelvés, le marquis de Florés et don Gonzalo de Córdoba. Ils étaient chargés d'étudier un rapport du Conseil des Indes portant sur le transfert de la ville vers un site plus commode, accompagné de documents écrits par l'archevêque et le vice-roi de México décrivant l'état lamentable de la cité. Les cinq membres du Conseil se déclarèrent tous en faveur du transfert, notamment le marquis de Gelvés, ancien vice-roi de Nouvelle-Espagne entre 1621 et $1624 .{ }^{19}$ Celui-ci disserta longuement sur les différentes possibilités offertes aux autorités royales. Sa connaissance du milieu lui permettait de ne pas être dupe des propositions faites par les différents groupes de pression représentés sur place. En effet, le transfert de toute une ville avait un impact très fort sur le marché foncier, et certains individus ("los poderosos") pouvaient essayer d'en tirer le plus grand profit possible: outre le fait que leurs maisons de campagne se trouvaient d'un seul coup situées au cœur d'un grand projet urbain, ils pouvaient revendre aux représentants de la Couronne et de la municipalité, au meilleur prix, les terres qui leur appartenaient (carte $\left.n .^{\circ} 2\right)$.

\section{Carte n. ${ }^{\circ}$ 2: Les projets de transfert de Mexico}

La technique utilisée par les habitants de México pour s'opposer au transfert est exposée en filigrane dans la fameuse Relación universal legitima y verdadera del sitio en que está fundada la muy noble, insigne y muy leal Ciudad de México..., publiée sur place en 1637. ${ }^{20}$ Tout part de la cédule royale du 19 mai 1631 qui ordonne d'examiner tous les lieux où le déplacement de la capitale pourrait se faire sans porter préjudice aux Espagnols obligés d'abandonner leurs maisons, ni aux Indiens à qui l'on risquait de confisquer les terres. Après avoir accusé réception du document et approuvé son contenu, selon la coutume, les échevins ont tout de suite engagé le fer avec l'autorité de la Couronne. Dans un premier temps, ils se contentèrent de citer des autorités incontestables, comme don Luis de Velasco, à qui la ville devait l'érection de la digue de San Lázaro (1556). En effet, dans une lettre datée du 16 septembre 1555 , le bon vice-roi écrivait à Charles

19 Archivo General de Simancas, Estado, 2649.

20 Cepeda, Fernando de; Carillo Fernando Alfonso; Álvarez Serrano. Juan: Relación universal legitima y verdadera del sitio en que está fundada la muy noble, insigne y muy leal Ciudad de México..., Centro de Estudios de Historia de México de CONDUMEX, México, 1983. 
Quint: "no tiene reparo la ciudad si no se mudase, y esto ya no se puede hazer, assi porque costaria dinero innumerable, como porque ni podrian, ni querrian los Indios entender en obra tan grande, y sin ellos no se puede hazer una casa, quanto mas mudar una tan grande ciudad". ${ }^{21}$ Un autre acteur fondamental de la vie coloniale, l'archevêque de Mexico, don Francisco Manso, fut mis à contribution, malgré ses réticences. Sollicité en octobre 1629 par le vice-roi marquis de Cerralvo pour exprimer son opinion sur le thème du transfert, il préconisa d'attendre la décrue des lacs avant d'entreprendre quoi que ce soit. ${ }^{22}$

Dans le même temps, et suivant une tactique éprouvée, les opposants au projet inondèrent littéralement, et sans jeu de mots, le conseil des Indes de lettres et de rapports destinés à fléchir la volonté de la Couronne. Les uns pressaient le roi d'accélérer les travaux de drainage, les autres tentaient de démontrer l'impossibilité du projet de transfert. C'est le cas des pères augustins qui, le 25 janvier 1630, signalèrent que "algunos avían llegado a pensar que se havía de mudar el sitio de tan grande lugar como es México, cosa que no se debe por ningún camino platicar". ${ }^{23}$ Cet enthousiasme n'était pas toujours communicatif, surtout quand, au fil des mois, les eaux tardèrent à se retirer. C'est pourquoi les échevins employèrent une dernière ruse: plutôt que de s'opposer directement à la volonté royale, ils décidèrent de la contourner en faisant d'abord remarquer que le coût du transfert, compte tenu des édifices à reconstruire, des impôts à reporter, des rentes perdues et des aides financières destinées à la population, serait phénoménal, comparé au prix du drainage des lacs.

En effet, la valeur de México (immobilier et mobilier compris) peut varier selon les sources mais, en 1603, le marquis de Montesclaros avançait déjà le chiffre de quatre-vingt-six millions de pesos. En 1629, une autre estimation atteignait les cent vingt-cinq millions de pesos. ${ }^{24}$ Quant aux auteurs de la Relación universal..., ils proposaient cinquante millions, alors que les frais des travaux de drainage étaient évalués à quatre millions. Quelle que fût la valeur réelle de la cité, le roi avait donc tout intérêt à laisser les ingénieurs achever leur travail, plutôt que de se lancer dans une aventure aux conséquences économiques difficiles à calculer. Comme le rappelait fort justement Alexandre de Humboldt dans son Ensayo político

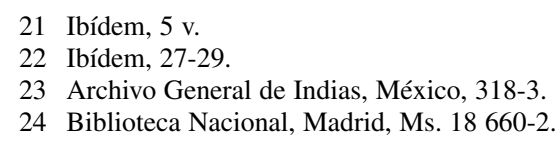




\section{ALAIN MUSSET}

sobre el reino de la Nueva España, publié pour la première fois en 1808: "Parece que se ignoraba en Madrid que la capital de un reino construida ya 88 años antes, no es un campo volante que se muda cuando se quiere". ${ }^{25}$ Pour mieux prouver leur bonne foi et leur désir de suivre les conseils de la Couronne, les membres du conseil municipal s'engagèrent à examiner la proposition de déplacement, et même de l'appliquer, si les travaux de drainage ne donnaient pas de bons résultats. Cette condition explique pourquoi, à plusieurs reprises, les autorités de Mexico se sont empressées d'annoncer la fin victorieuse des travaux. Il ne s'agissait pas seulement de se réjouir parce que la capitale de la Nouvelle-Espagne était enfin sauvée des inondations, il fallait aussi convaincre les autorités royales du succès de l'entreprise, afin de les détourner de leur objectif initial: le déplacement de la ville.

\section{Le sol sacré de la "patrie"}

En effet, vouloir déplacer México contre la volonté de ses habitants, c'était compter sans l'esprit de la cité - son âme immortelle, pourrait-on dire. Quelques années après l'indépendance, le père Andrés Cavo transcrivait le discours d'un opposant au transfert afin de mettre en valeur la résistance des créoles mexicains devant un ordre injuste et humiliant, alors que les Aztèques n'avaient jamais reculé devant les inondations: “¿Cuales fueron las consecuencias de aquella desgracia? ¿Acaso abandonar la ciudad y trasladar la silla del reino de México a pasaje mas encumbrado? No por cierto, señores. Los mexicanos jamas se hubieran resuelto a este paso; amaban, como se debe amar, la patria". ${ }^{26}$ La Patrie: le mot est lâché. La résistance opiniâtre des créoles mexicains met en valeur la lutte, trop souvent occultée (ou bien limitée à des querelles administratives), des Espagnols installés en Amérique contre un pouvoir lointain qui ne prenait pas en compte leur spécificité.

De fait, pour la Couronne, le déplacement d'une ville n'était qu'un problème technique et financier, alors que pour ses habitants, il s'agissait d'une remise en cause profonde, d'un échec collectif et personnel, d'un

25 Humboldt, Alejandro de: Ensayo político sobre el reino de la Nueva España, Porrua, Sepan Cuantos 39, 1984, pág. 128

26 Rubio Mañe, J. Ignacio: Introducción al estudio de los virreyes de Nueva España, T. IV, Obras públicas y educación universitaria, UNAM, México, 1963, págs. 97-98. 
ultime renoncement. Car ce ne sont pas seulement des pierres qu'on abandonne: c'est aussi, et surtout, un lieu consacré, dédié à la plus grande gloire de Dieu, où reposent parfois les morts de plusieurs générations. Le souvenir des ancêtres, comme dans beaucoup d'autres civilisations, occupe une place centrale dans la mémoire collective des citadins venus de la péninsule ibérique. De la même manière, au cours de la dernière guerre punique (149 av. J.-C.), c'est pour éviter de laisser leurs morts sans surveillance que les Carthaginois ont refusé l'offre romaine de s'exiler, préférant périr en compagnie de leurs dieux: "Née de la mer, la cité était demeurée essentiellement un port et elle ne pouvait respirer que devant la mer. Comment eût-elle abandonnée ses morts, son tophet, témoin de tous ses sacrifices, et les sanctuaires de ses dieux? Les Carthaginois décidèrent donc enfin de se défendre jusqu'à la mort" ${ }^{27}$ Afin de justifier leur choix, les adversaires du déplacement de Chillán firent eux aussi appel à la corde sensible de leurs concitoyens. Dans une lettre adressée à l'intendant de la province, quelques mois après le tremblement de terre qui avait jeté au sol presque toutes les maisons construites après le précédent transfert de 1751, Juan Antonio Zuñiga évoquait ainsi l'affection des habitants pour la ville qui les avait vus naître et dans laquelle ils avaient toujours vécu: "hasta la melancolia es un plaser y se exita al mirar el suelo donde se salvó la vida el 20 de febrero de 835". ${ }^{28}$

Encore au début du XIX ${ }^{\mathrm{e}}$ siècle, il ne fallait pas badiner avec la religion. Devant la détermination des autorités locales, décidées à hâter le départ des récalcitrants, le curé du Viejo Chillán entreprit de défendre coûte que coûte les intérêts de sa paroisse, en invoquant la loi qui autorisait les habitants à rester sur place malgré la décision officielle du transfert. Il en fut même réduit à soutenir ses vieux adversaires du clergé régulier, dont on avait adjugé au plus offrant les terrains et les biens immobiliers. D'après lui, la colère des sinistrés avait atteint son comble quand on avait rasé l'église paroissiale (bâtie aux frais de la communauté), pour vendre la parcelle vingt pesos à un simple particulier. On avait de la sorte touché au caractère sacré du sol urbain, dont l'usage ne se limitait pas seulement à des activités profanes, mais aussi aux pratiques religieuses et aux besoins du culte. Indigné, le saint homme fit remarquer à l'intendant que la municipalité n'avait reculé devant aucune manœuvre pour décourager les habitants pág. 223.

27 Decret, François: Carthage ou l'empire de la mer, Le Seuil, Points-Histoire, Paris, 1977, 28 Archivo Nacional, Santiago de Chile,Varios, vol. 257, 180, 379. 
du Viejo Chillán: fermeture des rues, dépavage de la place centrale, démolition du marché... Pourtant, rien ne les avait autant affectés que la destruction de leurs lieux de culte. C'est pourquoi, avant toute chose, ses ouailles réclamaient le rétablissement de l'église, qui seule pouvait leur garantir de ne pas se transformer en un troupeau de brutes privées de nourritures spirituelles. ${ }^{29}$

De fait, la mort d'une ville était définitivement consommée quand les cloches de l'église étaient retirées pour être transportées en un autre lieu. C'est ce qui arriva à la malheureuse cité de Zamora (Équateur), en 1735, quand ses habitants décidèrent d'abandonner le site qu'ils occupaient depuis 1663. Le transfert organisé à l'époque, afin de rendre vie à l'ancienne capitale de la province, avait échoué. Réduits à une poignée de chrétiens entourés d'Indiens hostiles, les Espagnols regagnèrent la cité de Loja, qui leur offrait de meilleures garanties de bien-être et de sécurité, emportant avec eux les cloches de la paroisse. Dans un autre contexte, Antonio de Ciudad Real évoquait le cas de la petite ville de San Miguel (El Salvador), peuplée en 1586 d'une trentaine de familles d'Espagnols. ${ }^{30}$ Alors que, pour la troisième fois depuis sa fondation, le feu venait de dévaster les fragiles maisons de bois, aux toits de paille, qui composaient l'agglomération, les autorités civiles, suivies par de nombreux habitants, avaient décidé de s'installer ailleurs. De manière symbolique et solennelle, ils avaient alors emporté les cloches des couvents et des églises, au grand dam des partisans de la reconstruction des édifices détruits qui protestèrent en vain contre ce coup porté à l'essence même de leur cité. En 1684, pour en finir avec la fiction juridique de la ville de Caloto (Colombie), qui n'avait plus de ville que le nom, l'évêque de Popayan suggéra d'emporter les cloches de l'église, considérées comme "una gran reliquia", et de les entreposer dans sa cathédrale.$^{31} \mathrm{Il}$ espérait de cette manière convaincre les habitants accrochés à leur rêve de grandeur qu'il était temps pour eux d'abandonner une cité moribonde, désormais privée de ses symboles les plus sacrés.

À un niveau plus élevé dans la hiérarchie ecclésiastique, le sort de la Antigua Guatemala est resté suspendu durant plusieurs années au maintien sur place de la paroisse du Sagrario, réservée à l'élite des familles d'origine espagnole. Malgré la longue résistance des ordres religieux et du clergé

29 Archivo Nacional, Santiago de Chile,Varios, vol. 257, 180, 386 v.

30 Ciudad Real, Antonio de: Tratado curioso y docto de las grandezas de Nueva España, UNAM, México, 1993 (vers 1584), pág. 241.

31 Archivo General de Indias, Quito, 215, 3, f. 214. 
séculier, et plus particulièrement de l'archevêque Cortés y Larraz, la cathédrale de la Nueva Guatemala de la Asunción fut consacrée le 22 novembre 1779. La perte du siège de l'archevêché, source inestimable de prestige et de pouvoir, marqua la fin réelle de la ville ancienne, même si quelques familles refusèrent jusqu'au bout d'obéir aux injonctions du Capitaine général.

\section{La résistance des Créoles}

Dès la fin du XVI ${ }^{e}$ siècle, alors que le clivage entre péninsulaires et créoles n'était pas encore complètement enraciné dans les mœurs de la société coloniale, la tension entre les deux groupes était déjà palpable. À de nombreuses reprises, les décisions de la Couronne ont été contestées et, sans remettre en cause de manière directe l'autorité du roi ou de ses représentants, les conquérants ou leurs héritiers ont tenté de contourner les décisions prises à Madrid, à Valladolid ou à l'Escurial. Il ne s'agissait plus de divisions internes à la communauté espagnole, mais bien d'une coupure entre un pouvoir lointain et ses administrés, entre la Couronne espagnole et ses sujets du Nouveau Monde - avant la lettre, on pourrait invoquer l'émergence d'un combat latent entre le global et le local. Ainsi, la lenteur du déplacement de Nombre de Dios vers Portobelo, entre 1573 et 1596, s'explique en grande partie parce que les commerçants et les propriétaires de bateaux, installés dans une ville pourtant détruite à deux reprises par sir Francis Drake, ont longtemps traîné des pieds pour rejoindre le site choisi par les juges de l'audience de Panama. L'un de ses membres, le docteur Diego de Villanueva Zapata, ne pouvait que constater, à son grand regret, que "Los vezinos han gustado poco desta mudanza". ${ }^{32}$

En outre, avec le temps, la position des différents groupes de pression pouvait évoluer. Les échevins de Guatemala, qui étaient les premiers à réclamer l'abandon du site en 1717, entrèrent en résistance en 1773 et s'opposèrent au Capitaine général, don Martín de Mayorga, accusé de vouloir brimer les populations autochtones. Ils rappelèrent au Roi qu'on n'abandonnait pas aussi facilement la capitale d'une grande province et que les Espagnols venus de la métropole devaient respecter l'opinion des créoles qui avaient toujours vécu sur les terres conquises par leurs ancêtres:

32 Archivo General de Indias, Panamá, R. 12, 71. 


\section{ALAIN MUSSET}

"Finalmente, el concepto que preocupa al gobierno de que es precissoo aniquilar a Guatemala para que esta ciudad se crie, lo juzga este cavildo absoutamente imberso y equivocado, discurriendo en el asumpto con total abstraccion del Patriotismo y sólo gobernado por lo que le inspira la razón y el zelo por el mejor servicio de VM y Bien del Publico". ${ }^{33}$ C'est donc la Couronne qui, en 1775, ordonna le transfert de Santiago de Guatemala vers son nouveau site, malgré l'opposition d'une grande partie des habitants, comme elle avait voulu le faire un siècle et demi plus tôt pour Mexico. Une première rupture fut alors consommée entre les trois éléments qui garantissaient l'existence et la pérennité de la cité espagnole en Amérique (le Roi, l'Église, la Municipalité). On peut considérer que, d'une certaine manière, ce transfert a servi de fondement aux revendications indépendantistes des créoles guatémaltèques qui, dans les masses de lettres et de rapports qu'ils envoyaient au Conseil des Indes à l'époque du transfert, usaient et abusaient du mot "Patrie" pour désigner leur ville en ruine.

Entre 1773 et 1775 , la fracture s'est rapidement élargie entre, d'une part, les principaux fonctionnaires de la Couronne, avec à leur tête le Capitaine général don Martin de Mayorga, et, d'autre part, les habitants hostiles au transfert. Les premiers à quitter la ville en ruine furent les employés du Trésor royal. Dès le 12 août, ils s'installèrent dans le campement provisoire du valle de la Ermita. Quelques jours plus tard, ils accusèrent réception des sceaux et des coins conservés à l'hôtel des Monnaies. La mesure était plus que symbolique: elle marquait de manière officielle la passation des pouvoirs entre l'ancienne et la future capitale de la Capitainerie générale du Guatemala. Malgré les rappels répétés de don Martín de Mayorga, les échevins traînèrent tous des pieds pour quitter leurs demeures, trouvant sans cesse de nouveaux prétextes pour retarder leur départ ou pour ralentir les préparatifs du déménagement. Pour vaincre la résistance des plus opiniâtres, il fallut employer les grands moyens et pratiquer la politique de la terre brûlée. En 1774, le représentant du roi décida d'imposer par la force le départ des habitants de Guatemala, interdisant à quiconque de rester sur place pour éviter le maintien ou la survie d'une ville, d'un village ou d'un simple lieu dit. ${ }^{34}$

On procéda alors au démantèlement des immeubles touchés par le séisme, sous prétexte d'en extraire les matériaux nécessaires à la construction de la ville nouvelle: pierres taillées, poutres, fers forgés, balcons, por-

33 Archivo General de Centro-América, Guatemala, A1.10, 2 444, 18746.

34 Ibídem. 
tes, volets... Par la même occasion, on s'attaqua aussi aux édifices encore intacts. Cette saignée, voulue et dirigée par le Capitaine général, se transforma en hémorragie. L'ancienne capitale devint une immense carrière à ciel ouvert. Chaque jour, de lourds convois extrayaient le matériel nécessaire aux constructions de la ville nouvelle. Le 28 juillet 1777, le capitaine général publia un décret qui sonna comme une véritable proclamation de guerre à mort contre la Antigua Guatemala: "he resuelto declarar como por el presente declaro, que la población de Goathemala, debe quedar enteramente extinguida, y asolada, dentro del preciso término de un año contado desde la fecha de este "..35

À partir du mois d'avril 1779, son successeur, don Matías de Gálvez, poursuivit la même politique en menaçant de prison tous ceux qui refuseraient de quitter la ville, sans distinction de race, de sexe ou de condition hommes et femmes, riches ou pauvres, Blancs, Noirs ou Mulâtres. De telles mesures suscitèrent la vive réaction des autorités religieuses qui, à l'initiative de l'archevêque Cortés y Larraz, prirent à nouveau fait et cause pour les habitants de la Antigua. Cependant, le départ de l'ecclésiastique pour l'Espagne, en septembre 1779, leur fit perdre un avocat puissant et respecté. Bien que d'origine péninsulaire, il avait su s'attirer la sympathie des populations locales en s'opposant de manière presque systématique aux ordres des capitaines généraux, considérés comme la figure emblématique d'un pouvoir lointain, qui ne pouvait ou ne voulait pas comprendre les traditions et les particularités du monde américain.

Les mêmes clivages apparaissent de manière explicite dans le dossier concernant le projet de déplacement de la ciudad de Londres vers le site de Catamarca (Argentine), en 1675-1678. Les autorités locales s'estimaient lésées par la décision du gouverneur, nommé par le roi, qui prétendait les priver d'une partie de leur territoire, de leurs prérogatives administratives et judiciaires, mais aussi de leur main-d'œuvre indigène. Elles adressèrent donc à la Couronne un abondant courrier pour protester contre la politique menée en son nom dans la province. Dans cette affaire, deux partis s'opposaient clairement: d'un côté, le conseil municipal qui représentait les intérêts des Créoles; de l'autre, les gouverneurs et les fonctionnaires de l'administration royale, considérés comme des étrangers incapables de répondre aux attentes de la population née en Amérique. Comme le rappelaient les

35 Pardo, J. Joaquin: Efemérides de la Antigua Guatemala (1541-1779), AGCA-BNGCNPAG-IAH, Guatemala, 1984, pág. 213. 
échevins de la Rioja: "Tambien suponemos que los gobernadores que vienen en vuestro real nombre assisten en el gobierno, unos tres, otros cinco o seis años, y discurriendo en las materias, conforme los sucessos que en sus gobiernos acaessen, que para conocimiento de gentíos, nuevas costumbres, tratos que nunca vieron, no son bastantes años para la experiencia, que lo que no vieron en el corto tiempo de sus gobiernos, lo hemos visto antes y después, los que somos nacidos en estas tierras". ${ }^{36}$

Le "los que somos nacidos en estas tierras" marque la différence profonde ressentie par les créoles vis à vis de cette cohorte de péninsulaires arrogants qui ne faisaient que traverser le Nouveau Monde - véritables mercenaires d'État dont le seul désir était de regagner l'Europe une fois fortune faite. Dans ces conditions, le meilleur moyen d'obtenir gain de cause était de proclamer haut et fort sa fidélité au roi et de montrer que les revendications locales n'avaient pas pour but de satisfaire des intérêts particuliers, mais bien de servir les desseins de la Couronne. Les échevins hostiles au projet tentèrent donc d'attendrir les membres du Conseil des Indes en leur montrant comment, depuis les premiers temps de la conquête, les gouverneurs de la province avaient, par maladresse ou par calcul, maltraité leurs administrés et, de manière indirecte, porté préjudice au Trésor royal. La quatrième tentative de restauration de la ville de Londres ne faisait que suivre la même ligne, puisque, pour mener à bien cette opération, on menaçait de démembrer les communes voisines, La Rioja et Tucuman. Le tout se solderait, selon les membres du conseil municipal de La Rioja, par la perte de leurs meilleures terres agricoles, au profit d'une ville nouvelle dont l'avenir était loin d'être assuré. La baisse des revenus entrainée par cette décision arbitraire allait grever les finances de la commune. Par contrecoup, c'est tout le système politique de la zone qui serait affecté, puisque les vecinos n'auraient plus les moyens d'assumer leur rang et de faire face à leurs responsabilités financières - plus particulièrement vis à vis de l'Église, puisqu'ils avaient à leur charge l'entretien de quatre couvents et de deux paroisses (dont l'évêché).

Leur discours oppose de manière systématique les trois forces en présence: le roi, respecté mais trop lointain; les gouverneurs, qualifiés de "zélés", mais qui ignorent tout des terres et des hommes placés sous leur tutelle; les habitants "nobles et loyaux", regroupés dans une "ville loyale", et représentés par un conseil municipal "prosterné aux pieds de votre

36 Archivo General de Indias, Charcas, 23, R.7, N.71b (bis), f. 29. 
majesté". ${ }^{37}$ Malgré ces protestations de fidélité et de loyauté, on sent poindre chez les descendants des conquistadors une grande amertume. On ne les a pas compris. On les a dédaignés. Les représentants de la Couronne méprisent leurs avis, ignorent leurs besoins, balayent d'un revers de la main leurs conseils ou leurs objections. Pourtant, en sujets dévoués, ils n'ont jamais hésité à verser leur sang pour l'honneur, la gloire et le plus grand profit du Roi, leur "seigneur naturel". En ce qui concerne les gouverneurs, ces parvenus et ces "tard venus", c'était une autre affaire, car on ne devait rien à des gens qui voulaient vous opprimer: "Señor, si la dicha ciudad tan leal a vuestra corona y con tanto gasto de sus hassiendas, sangre y vidas, os ha servido con tan gloriosos desenpeños de vuestras reales armas (lo que Dios no permitirá), os ubiera faltado a la lealtad que os debe como a su Rey y Señor natural, pudieran vuestros gobernadores executar mayor castigo que privarla de las [mercedes], de los premios, de los favores que le havéis hecho, y dejarla sin medios para su sustento y que perezca y se deshaga a manos de su desanparo y nessedidad: este castigo, Señor, es el que quieren darle vuestros gobernadores". ${ }^{38}$

En insistant sur les services que, dans le passé, ils avaient rendus à la Couronne, les habitants de La Rioja défiaient donc ouvertement ses représentants actuels. De manière indirecte, et peut-être inconsciente, ils remettaient en cause la légitimité d'un pouvoir qui ne les appréciait pas à leur juste valeur alors qu'une bonne partie de sa force reposait sur leur fidélité.

\section{Conclusion}

Pour autant, la crise qui sert de révélateur aux fractures de la société coloniale ne se limite pas aux débats provoqués par le choix du déplacement ou du maintien dans les lieux. Comme dans toute bonne tragédie, il faut au moins trois actes pour aller au bout de l'action: le désastre qui pose la question du déplacement; les discussions où s'opposent les différents points de vue; les querelles qui concernent le choix du nouveau site. À chaque étape du processus, les discours enflammés, les lettres personnelles, les pétitions collectives, les compte rendus de réunion, les bandos municipales o las reales cédulas éclairent d'un jour nouveau les racines politiques et religieuses de la cité espagnole. En effet, pour les partisans du transfert

37 Ibídem, N.71, .15.

38 Ibídem, N.71b (bis), 21. 
comme pour ceux de la reconstruction la ville n'est pas seulement un assemblage plus ou moins harmonieux de pierres, de poutres et de briques où se rassemblent des individus, des familles et des groupes sociaux: la ville est avant tout un symbole dont le caractère sacré assure la cohésion de la communauté et légitime son existence.

C'est pourquoi, en 1717, les adversaires du déplacement de la ciudad de Santiago de los Caballeros de Guatemala font bien la distinction entre le parcours individuel des habitants et le destin de la cité détruite: "Y así los temblores, que son causa suficiente para que los particulares que quisiesen muden sitio, no es suficiente para la mudanza de una ciudad. La razón de Estado de una ciudad no consiste en la tierra ni el suelo, sino que tiene varas mas firmes y mas profundos fundamentos, que son el bien público y utilidad común de todo este dilatadísimo reyno; y no se puede mover sino es atropellando con la autoridad del Rey Nuestro Señor, cuyo es propio y privativo el eregir ciudades en sus reynos y señarlas por residencias de sus Reales Audiencias y chancillerías que representen su Real Persona; ni se puede mudar a otro sitio el cabildo eclesiástico y cathedra episcopal, sino es usurpándose la autoridad del sumo Pontífice, a que únicamente toca el eregir o mudar las ciudades que sean cathedras y sedes episcopales; ni es posible mudar de este sitio los alcaldes y cabildo secular, si no es dejándolo sin representación, especie, ni aparencia de ciudad". ${ }^{39}$

Pour les deux groupes qui s'affrontent, l'essentiel est d'assurer la continuité du fait urbain, sur place ou dans un autre lieu, car l' "urbs" n'est rien sans la "civitas". C'est ce qu'affirment les habitants de León (Nicaragua), quand ils s'éloignent du volcan Momotombo pour s'installer près de la communauté indigène de Subtiava. L'acte notarié rédigé à cette occasion stipule que si la ville a changé de place, il s'agit toujours de la même ville: "abiendo traçado y fundado esta dicha ciudad en este sitio en nombre de su magestad, laqual dicha ciudad es la que estava poblada seis leguas desta dicha ciudad junto a el volcan y laguna". ${ }^{40}$ Aux yeux des habitants convaincus de leur bon droit, le déplacement n'est donc pas synonyme de mort: c'est une renaissance qui, comme toutes les naissances, doit se faire dans la douleur.

39 Biblioteca Nacional, Madrid, Ms 3 534, 55 v. et 57

40 Archivo General de Indias, Guatemala, 43, 26, 26 v. 